\title{
HON ou comment garantir la qualité des informations médicales sur internet
}

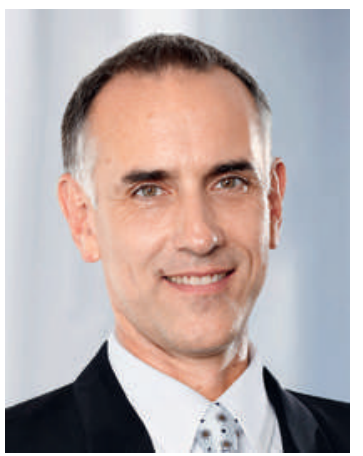

En dépit de sa forte valeur ajoutée, le service d'envergure internationale dont il est question ici est à tort mal connu. Elaboré par une organisation non gouvernementale (ONG), sise à Genève et créée en 1995 sous la forme d'une fondation, le label Health on the Net, également dénommé HON, certifie la qualité des sites dédiés à la santé. Sa mission consiste à aider à reconnaître les informations médicales fiables, compréhensibles et pertinentes divulguées sur internet.

Aujourd'hui, de plus en plus de patients s'informent par internet du tableau clinique d'une maladie et des possibilités de traitement avec, pour les profanes, toute la difficulté de savoir si une information est objective, fiable et encore actuelle. Dans ce contexte, un service garantissant la qualité du contenu des sites internet revêt d'autant plus d'importance. Il s'agit notamment d'assurer la transparence sur l'origine des informations et sur le financement de l'offre. Par ailleurs, les données mises en ligne devraient être vérifiées par une instance indépendante.

\section{Pour les patients, internet est souvent la première source $d$ 'informations lors de questions médicales.}

Pour certifier la fiabilité et la crédibilité des sources d'informations en ligne selon des critères formels, la fondation a établi un code de conduite, le HONcode, qui impose huit principes à respecter dont, entre autres, offrir des informations véridiques, ne pas entraver la relation médecin-patient et préserver la confidentialité des données personnelles. Avant de délivrer un certificat, la fondation HON contrôle si les critères définis sont respectés lors de la première certification et procède ensuite à des vérifications régulières. Le feu vert est à chaque fois donné par un conseil médical neutre.

En Suisse, la situation en la matière est actuellement la suivante. Le rapport «Portail de santé publique» publié le 26 jan- vier 2012 par eHealth Suisse (Confédération et cantons) relève l'importance de garantir la qualité des sites internet publics et privés consacrés à la santé. En mars 2012, la conseillère nationale Edith Graf-Litscher a demandé au Conseil fédéral quel crédit celui-ci accordait au label de qualité HON dans le but de garantir la fiabilité des informations médicales particulièrement sensibles divulguées sur internet. Le Conseil fédéral a répondu qu'en 2009 déjà, le comité de pilotage d'eHealth

\section{Parce que primordiale, la garantie de la qualité des informations médicales doit être encouragée.}

Suisse avait proposé à la Confédération et aux cantons de promouvoir le travail de sensibilisation en vue d'assurer la qualité des informations médicales et sa mise en œuvre. En janvier 2012, ce comité a renouvelé sa recommandation et a invité les prestataires de sites internet consacrés à la santé à faire certifier leurs offres, par exemple, avec le label de la fondation HON.

Le HONcode, le label de qualité le plus répandu dans notre pays et dans le monde pour les informations médicales en ligne, a permis jusqu'à présent de certifier près de 310 sites de santé en Suisse; en France, où la certification HON bénéficie du soutien financier de la Haute autorité de la santé (HAS), pas moins de 1200 sites ont été labellisés.

Au plan fédéral, la législation actuelle ne comporte aucune base permettant de soutenir financièrement de tels labels. Cependant, fin mai 2013, la Confédération a organisé une conférence des donateurs dans le but de créer un cadre optimal pour la mise en œuvre de la stratégie eHealth Suisse. Le Conseil fédéral a ainsi voulu contribuer au renforcement du socle financier alimenté par les acteurs privés et les cantons au cours des prochaines années, afin que la fondation HON puisse poursuivre son travail. Comme la Toile représente aujourd'hui une source prépondérante de renseignements pour les patients, il convient de garantir la qualité à long terme des informations médicales en ligne et de promouvoir le code de conduite HONcode.

Dr Gert Printzen, membre du Comité central de la FMH, responsable du domaine Informatique médicale et eHealth 